\title{
Positron interactions with nitrogen and oxygen molecules: elastic, inelastic and total cross sections ${ }^{\star}$
}

\author{
Lilian Ellis-Gibbings ${ }^{1}$, Francisco Blanco ${ }^{2}$, and Gustavo García ${ }^{3, a}$ \\ 1 Chemistry department, University College London, 20 Gordon St, London WC1H 0AJ, UK \\ 2 Departamento de Física Atómica, Molecular y Nuclear, Universidad Complutense de Madrid, Madrid 28040, Spain \\ 3 Instituto de Física Fundamental, Consejo Superior de Investigaciones Científicas (CSIC), Calle Serrano 113bis, Madrid 28003, \\ Spain
}

Received 17 September 2019 / Received in final form 21 November 2019

Published online 24 December 2019

(C) The Author(s) 2019. This article is published with open access at Springerlink.com

\begin{abstract}
Positron scattering cross sections, used for modelling particle transport in various media, are difficult to gather experimentally. As such, various cross section calculation methods have been developed to varying accuracy. The IAM-SCAR+I method has been improved upon recently to fulfil the optical theorem and the results for two important simple molecules, $\mathrm{N}_{2}$ and $\mathrm{O}_{2}$, are presented here. These results are compared to literature and our findings are comparable in most impact energy ranges.
\end{abstract}

\section{Introduction}

The simulation of positron tracks in various media is an important field for the biomedical community [1]. Positron emission tomography [2] (PET) is the most well-known medical use of positron emitters, however positron dosimetry for ion beam therapy [3] is a growing clinical practice. It allows higher accuracy in tracking energy deposition via the production of positron emitters through nuclear inelastic collisions. Positrons used in PET and ion dosimetry exhibit a wide range of energies, with an average energy of hundreds of $\mathrm{keV}$ [4] and up to several $\mathrm{MeV}$. The positron typically emits its detectable gamma photons near the end of its transport track, and there the kinetic energy could have to below $100 \mathrm{eV}$.

Monte Carlo modeling procedures capable of tracking positrons in various media require accurate interaction cross section data [5] over a broad energy range. This includes but is not limited to the elastic and inelastic scattering, ionization, electronic excitation, positronium formation and annihilation down to $0 \mathrm{eV}$. A high number of electron ionizations necessitates tracking of the secondary electrons within the same code, using electron interaction data.

A recently released database for positron scattering from molecular targets [6] has a reliable compilation of integral and differential cross sections for both $\mathrm{N}_{2}$ and $\mathrm{O}_{2}$.

\footnotetext{
* Contribution to the Topical Issue "Low-Energy Positron and Positronium Physics and Electron-Molecule Collisions and Swarms (POSMOL 2019)", edited by Michael Brunger, David Cassidy, Saša Dujko, Dragana Marić, Joan Marler, James Sullivan, Juraj Fedor.

a e-mail: g.garcia@csic.es
}

This database covers the projectile kinetic energy range of 0.11 to $750 \mathrm{eV}$ for $\mathrm{N}_{2}$ and 0.10 to $600 \mathrm{eV}$ for $\mathrm{O}_{2}$. This compilation of data allows us to benchmark any new features of a cross section calculation against the current literature. As antiparticles, positrons present with experimental difficulty in achieving high beam currents of precise energies, as such most positron experiments are restricted to particular energy ranges and spreads [7]. For calculation, annihilation and positronium formation present the greatest challenges to realistic interaction cross sections. The energy range necessary to simulate positron transport for medical purposes crosses the region from which the First Born Approximation (FBA) applies $(>10 \mathrm{keV})$ to energies where it overestimates the cross section - as such individual calculation methods tend to have regions of higher and lower accuracy. Simulating the path of the positrons, their angular distribution, energy deposition and ranges, requires the differential (both energy transferred and scattering angle) and integral cross sections in each material. Within the inelastic cross section the formation of positronium must be treated as one of the recourses towards annihilation, dependent on the impact energy [8].

At high energies electron scattering cross section values are widely used in place of separately calculated positron cross sections [9]. This is justified by the scattering interaction being understood to be predominantly a static interaction at these energies. However, it was shown [8] that at energies as high as $10 \mathrm{keV}$ the polarization of the target introduces a systematic difference in the calculated cross sections for electrons and positrons. As such the positron scattering total cross sections for molecules tend to be lower in intensity than those of electrons due to the combination of signs between the polarization potential and the static potential, being the same for electrons and opposite for positrons. 
Recently headway has been made in approximate calculations on atomic and molecular cross sections for positron scattering. In a 2016 paper by Singh et al. [10] a modified version of the spherical complex optical potential (SCOP) formalism has been used to provide positron total cross sections from 1 to $5000 \mathrm{eV}$, and these follow the low energy phenomena quite well. As the field grows towards accuracy and consistency, it is imperative to compare the different methods used for achieving these results. The independent atom model with screening corrected additivity rule plus interference terms (IAM-SCAR+I) method of our group has also been improved, with the introduction of a screened interference term [11] including more realistic scattering processes in the results. In prior iterations of the IAM-SCAR method a scaling factor was used to account for a discrepancy with the optical theorem. This interference term has the added benefit of embedding consistency between the calculation of differential and integral cross sections, and thus validating the optical theorem within the method $[11,12]$.

In this article we produce new positron elastic, inelastic, differential and integral scattering cross sections for the $\mathrm{N}_{2}$ molecule, and updated results for the $\mathrm{O}_{2}$ molecule, using our IAM-SCAR+I method for molecular cross sections. These are compared to literature, including those more recently calculated by Singh [10]. First we introduce our method, IAM-SCAR+I, explained in detail in other publications [11-13] - an advancement on the IAM-SCAR method pioneered by Garcia and Blanco $[14,15]$ in the early 2000s. Second follows a detailed comparison with the existing positron scattering data [6,16-19]. Comments on the methods and comparisons are made and followed by concluding remarks.

\section{Calculation procedure}

\subsection{Optical potential method}

The IAM-SCAR+I method for the calculation of positron scattering cross sections has been improved in 2016, as outlined in our paper from Blanco et al. [8]. This method builds on our previous work $[4,11,12,14,15,20-24]$ for both electrons and positrons, and has been used successfully in the past for biologically relevant molecules such as water [8,25], pyrimidine [26-28], pyridine [29], benzene [30], diatomic oxygen [24,31], tetrahydrofuran [32], macromolecules [21] and many more, typically in the range of 0.1 to $10000 \mathrm{eV}$ incident energy.

IAM-SCAR + I relies on the optical potential method with a local complex potential representing each atomic scattering center, according to the equation:

$$
V(r)=V_{s}(r)+V_{p}(r)-i V_{a}(r)
$$

where $V_{s}(r)+V_{p}(r)$ is the real, or elastic scattering part of the optical potential, and $i V_{a}(r)$ is the imaginary absorption potential, accounting for the inelastic scattering channels. $V_{s}(r)$ is the static potential, describing the interaction between the positron and the atomic charge density. In contrast to the case of electron scattering by atoms, positrons are attracted by the target electrons and repelled by the nuclei. We formulate this term by a derivation of the Hartree-Fock atomic wavefunctions analogously to the work of Reid and Wadehra [33].

$V_{p}(r)$ is the polarization potential, giving the polarization (distortion) of the electron cloud of the molecule with the approach of the incoming positron. It is the sum of a dipole and quadrupole potential calculated with the polarized-orbital method by determining the first-order corrections of the atomic orbitals due to a fixed charge field [34]. This method is based off that proposed by McEachran et al. [34] for noble gases, from which we take the results for Neon and adjust in order to give the known dipole polarizability of each atom. For $\mathrm{O}$ and $\mathrm{N}$ the dipole plus quadrupole polarized orbital potentials of $\mathrm{Ne}$ (accurate against measurement [35]), are scaled so that they fit to the calculations of $\mathrm{N}$ and $\mathrm{O}$ presented in Reinsch and Meyer [36] and Werner and Meyer [37]. This method has been previously introduced and tested and was shown to produce good elastic scattering cross sections for positron impact $[24,29]$, as well as reproducing the expectation value of the orbital radius for various oxygen orbitals [24].

Note that another major difference compared to the scattering potentials of electrons is that the exchange term between the incoming electron and the target electrons is excluded as these effects do not occur with positrons. All $V_{a}(r)>0$ describe the "absorption" processes-i.e. the inelastic processes of excitation, ionization and positronium formation $(\mathrm{Ps})$ and require careful treatment. We use a scheme modified from that proposed again by Reid and Wadehra [33], assuming the target electrons can be considered as a quasi-free electron cloud with which the incoming particles undergo binary collisions. The threshold energy is carefully designed to include Ps formation as follows.

Our treatment of Ps formation has been outlined in detail previously [8]. In brief we maintain the energy dependent threshold $\Delta(E)$, by necessity coinciding with the well known Ps formation threshold of $\Delta_{\mathrm{p}}=\mathrm{I}-6.8 \mathrm{eV}$, (where $\mathrm{I}=$ ionization threshold) for lower energies and the lowest optically allowed excitation transition $\Delta$ for higher impact energies where positronium formation is unlikely. The improvement is equation (2), detailing the smooth transition in threshold energy from low to high impact energy:

$$
\Delta(E)=\Delta-\left(\Delta-\Delta_{p}\right) /\left[1+\left(\frac{E}{3 I}-1\right)^{2}\right] .
$$

When integral cross sections for different inelastic processes (positronium formation, electronic excitation and ionization) are desired, these are calculated simply by fixing the threshold for each process and running the calculation with each threshold. A simple subtraction of one process from the others gives an approximation for that channel. When treating only the electronic excitation, the first optically allowed excitation state is taken as the threshold. The threshold for ionisation of the molecule is based on the ionisation thresholds for the individual atoms. It should be made clear that the cross sections for each constituent atom are calculated using the known 
ionisation, excitation, and positronium formation thresholds of that atom before the application of the screening corrected additivity rule. For diatomic molecules this puts the ionisation limits at $13.6 \mathrm{eV}$ for $\mathrm{O}_{2}$ and $14.5 \mathrm{eV}$ for $\mathrm{N}_{2}$.

Direct annihilation is also a known inelastic channel but as the cross sections are several orders of magnitude lower in intensity than the ionisation, no attempt is made to include it.

\subsection{Screening corrected additivity rule plus interference}

The most important contribution pertaining to the IAMSCAR + I method is its treatment of molecular scattering. As described previously [8,12], the formalism for the IAMSCAR $+\mathrm{I}$ uses the potentials described to represent the scattering from the constituent atoms. Then a screening corrected additivity rule and a screening corrected interference term are applied. In brief:

$$
F(\theta)=\sum_{\text {atoms }} f_{i}(\theta) \mathrm{e}^{i \mathbf{q} \cdot \mathbf{r}_{i}}
$$

where $F(\theta)$ is the molecular scattering amplitude, $q=$ $k_{f}-k_{i}$ is the momentum transfer, the atomic positions are given by $r_{i}$ and the atomic scattering amplitudes are $f_{i}(\theta)$. This builds multi-centre scattering amplitudes as a sum of the independent scattering from each atom. The bond distances are taken from the pubchem database of $3 \mathrm{D}$ molecular structures [38]. For $\mathrm{N}_{2}$, the bond length is $1.12 \AA$, and for $\mathrm{O}_{2}$, it is $1.232 \AA$.

The molecular differential cross section $\left(d \sigma_{\text {molecule }}^{\text {elastic }} / d \Omega\right)$ as a combination of the multi-centre atomic amplitudes is given by:

$$
\begin{aligned}
\frac{d \sigma_{\text {molecule }}^{\text {elastic }}}{\Omega} & =\sum_{i j} f_{i}(\theta) f_{j}^{*}(\theta) \frac{\sin q r_{i j}}{q r_{i j}} \\
& =\sum_{i}\left|f_{i}(\theta)\right|^{2}+\sum_{i \neq j} f_{i}(\theta) f_{j}^{*}(\theta) \frac{\sin q r_{i j}}{q r_{i j}}
\end{aligned}
$$

Here the interference term is defined as the second summation in equation (4). In this case $q \equiv|q|=2 k \sin \frac{\theta}{2}$ is the momentum transfer and $r_{i j}$ is the distance between atoms $i$ and $j$. By integrating equation (5) the corresponding molecular integral cross sections are represented by:

$$
\sigma_{\text {molecule }}^{\text {total }}=\sum_{\text {atoms }} s_{i} \sigma_{\text {atom i }}^{\text {total }}+\sigma^{\text {interference }} .
$$

Factor $s_{i}$ is a screening correction, reducing the contribution of each atom to the total molecular cross section $\left(0 \leq s_{i} \leq 1\right)$ based on the position of the atom within the molecule. The derivation of this screening factor has been expounded fully in several previous works [12,15,21], and for brevity the details are omitted here. This accounts for the fact that as the energy of the incoming particle decreases, the atomic cross sections overlap, so requiring a reduction of their relative contribution to the sum [15].
The integrated interference term is then represented by:

$$
\sigma_{\text {interference }} \equiv \int^{d} \Omega \sum_{i \neq j} v_{i j} s_{i} s_{j} f_{i}(\theta) f_{j}^{*}(\theta) \frac{\sin q r_{i j}}{q r_{i j}}
$$

where $v_{i j}=r_{i j}^{2} /\left(r_{i j}^{2}+\rho_{i j}^{2}\right)$ is a factor introduced to attenuate the interference terms in accordance to their length dimensional parameter $\rho$, where $\rho_{i j}=\max \left(\sqrt{\frac{\sigma_{i}}{\pi}}, \sqrt{\frac{\sigma_{j}}{\pi}}, \frac{1}{k}\right)$ and varies with momentum $k$. As shown in Blanco et al. [12], including interference terms in the calculation of both integral and differential cross sections for molecular targets eliminates the inconsistency between the differential and integral cross section values which is inherent to the IAM-SCAR method. This means that no additional normalization procedure is required by the $\mathrm{SCAR}+\mathrm{I}$ approach in order to fulfil the optical theorem. Adding this screened interference term increases the range of expected validity of the calculation method down to an impact energy of $0.1 \mathrm{eV}$, as seen with electrons [12].

\section{Results and discussion}

Present results for the IAM-SCAR + I positron scattering integral cross sections (total, elastic, and inelastic, including electronic excitation, direct ionization and positronium formation) for molecular nitrogen are shown in Table 1 and plotted in Figure 1, and for molecular oxygen shown in Table 2 and plotted in Figure 2. These are the first results presented for positrons with $\mathrm{N}_{2}$ using the IAM-SCAR+I method and the first for $\mathrm{O}_{2}$ including the interference terms of the method.

Together with the present calculation for positrons we have included in Figure 1 the corresponding electron scattering TCS calculated with the same IAM-SCAR+I method. TCS data for electron impact energies above $100 \mathrm{eV}$, taken from the evaluated database from the Lawrence Livermore National Laboratory [39] (LLNL), are also plotted.

In Figures 1 and 2 the electron scattering TCS is also shown for comparison. We don't consider the IAM$\mathrm{SCAR}+\mathrm{I}$ method accurate at energies below $1 \mathrm{eV}$ for either electron or positron scattering and no comments can be made here. Comparing the electron and positron TCS makes clear that the positron TCS is lower than the electron TCS up to the limit of our calculation. As mentioned above this difference is due to the different sign combination of the polarization and static potential for electrons and positrons. The difference is more than $20 \%$ for energies above $500 \mathrm{eV}$. The inelastic processes shown in Figure 1 are ionisation, electronic excitation and positronium formation. These are determined from their respective thresholds (see Sect. 2. Calculation procedure) and are summed to give the total inelastic cross sections, excluding the vibrational and rotational excitations. Positron CS are available for various excitation states of $\mathrm{N}_{2}$ [40-42], and ionization, positronium formation and excitation have been investigated up to $90 \mathrm{eV}$ experimentally for both $\mathrm{N}_{2}$ and $\mathrm{O}_{2}$ [43]. These latter measurements are compared to our calculations towards the end of this discussion. 
Table 1. $\mathrm{N}_{2}$ integral cross sections calculated by the IAMSCAR + I method.

\begin{tabular}{|c|c|c|c|c|c|}
\hline $\mathrm{E}(\mathrm{eV})$ & $\begin{array}{l}\text { Elastic } \\
\left(\times 10^{-20}\right. \\
\left.\mathrm{m}^{2}\right)\end{array}$ & $\begin{array}{l}\text { Ps formation } \\
\left(\times 10^{-20}\right. \\
\left.\mathrm{m}^{2}\right)\end{array}$ & $\begin{array}{l}\text { Ionisation } \\
\left(\times 10^{-20}\right. \\
\left.\mathrm{m}^{2}\right)\end{array}$ & $\begin{array}{l}\text { Excitation } \\
\left(\times 10^{-20}\right. \\
\left.\mathrm{m}^{2}\right)\end{array}$ & $\begin{array}{l}\text { p-TCS } \\
\left(\times 10^{-20}\right. \\
\left.\mathrm{m}^{2}\right)\end{array}$ \\
\hline 0.1 & 53.48 & 0 & 0 & 0 & 53.48 \\
\hline 0.15 & 43.4 & 0 & 0 & 0 & 43.4 \\
\hline 0.2 & 36.68 & 0 & 0 & 0 & 36.68 \\
\hline 0.3 & 27.496 & 0 & 0 & 0 & 27.496 \\
\hline 0.4 & 21.84 & 0 & 0 & 0 & 21.84 \\
\hline 0.5 & 17.976 & 0 & 0 & 0 & 17.976 \\
\hline 0.7 & 16.016 & 0 & 0 & 0 & 16.016 \\
\hline 1 & 14 & 0 & 0 & 0 & 14 \\
\hline 1.5 & 11.06 & 0 & 0 & 0 & 11.06 \\
\hline 2 & 9.296 & 0 & 0 & 0 & 9.296 \\
\hline 3 & 7.504 & 0 & 0 & 0 & 7.504 \\
\hline 4 & 6.58 & 0 & 0 & 0 & 6.58 \\
\hline 5 & 5.936 & 0 & 0 & 0 & 5.936 \\
\hline 7 & 5.04 & 0 & 0 & 0 & 5.04 \\
\hline 10 & 4.004 & 0.504 & 0 & 0 & 4.508 \\
\hline 15 & 2.884 & 2.41 & 0.01526 & 2.27874 & 7.56 \\
\hline 20 & 2.912 & 2.1 & 1.2544 & 3.2536 & 9.52 \\
\hline 30 & 3.304 & 1.428 & 3.416 & 2.464 & 10.612 \\
\hline 40 & 3.416 & 1.148 & 4.144 & 1.82 & 10.528 \\
\hline 50 & 3.304 & 0.952 & 4.312 & 1.456 & 10.052 \\
\hline 70 & 2.856 & 0.588 & 4.144 & 1.148 & 8.736 \\
\hline 100 & 2.268 & 0.252 & 3.696 & 0.952 & 7.168 \\
\hline 150 & 1.7248 & 0.084 & 3.052 & 0.812 & 5.684 \\
\hline 200 & 1.4196 & 0.056 & 2.6096 & 0.7224 & 4.788 \\
\hline 300 & 1.0668 & 0.014 & 2.0356 & 0.6048 & 3.724 \\
\hline 400 & 0.8652 & 0.0056 & 1.68 & 0.5208 & 3.08 \\
\hline 500 & 0.7336 & 0.0028 & 1.4336 & 0.462 & 2.632 \\
\hline 700 & 0.5656 & 0 & 1.12 & 0.3752 & 2.0608 \\
\hline 1000 & 0.4284 & 0 & 0.854 & 0.2912 & 1.5736 \\
\hline 2000 & 0.24752 & 0 & 0.4844 & 0.1736 & 0.9072 \\
\hline 3000 & 0.17808 & 0 & 0.3444 & 0.1232 & 0.6468 \\
\hline 4000 & 0.14056 & 0 & 0.2674 & 0.0994 & 0.5068 \\
\hline 5000 & 0.11648 & 0 & 0.21924 & 0.08036 & 0.4172 \\
\hline 7000 & 0.08708 & 0 & 0.16156 & 0.06104 & 0.3108 \\
\hline 10000 & 0.06356 & 0 & 0.1162 & 0.04452 & 0.22428 \\
\hline
\end{tabular}

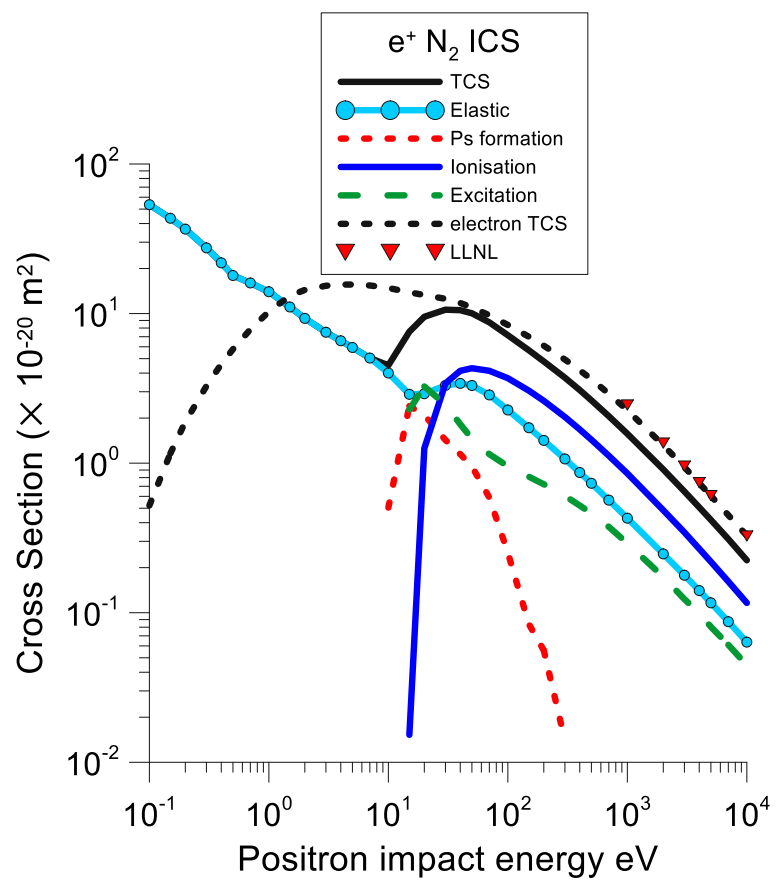

Fig. 1. Integral cross sections for $\mathrm{e}^{+}-\mathrm{N}_{2}$ calculated using the IAM-SCAR +I method. These include elastic and inelastic processes and the $\mathrm{e}^{-}-\mathrm{N}_{2}$ TCS calculated with the same method is shown for comparison. $\mathrm{e}^{-}-\mathrm{N}_{2}$ TCS from the Lawrence Livermore National Laboratory [39] (LLNL) included for comparison.
Table 2. $\mathrm{O}_{2}$ integral cross sections calculated by the IAM$\mathrm{SCAR}+\mathrm{I}$ method.

\begin{tabular}{|c|c|c|c|c|c|}
\hline $\mathrm{E}(\mathrm{eV})$ & $\begin{array}{l}\text { Elastic } \\
\left(\times 10^{-20}\right. \\
\left.\mathrm{m}^{2}\right)\end{array}$ & $\begin{array}{l}\text { Ps formation } \\
\left(\times 10^{-20}\right. \\
\left.\mathrm{m}^{2}\right)\end{array}$ & $\begin{array}{l}\text { Ionisation } \\
\left(\times 10^{-20}\right. \\
\left.\mathrm{m}^{2}\right)\end{array}$ & $\begin{array}{l}\text { Excitation } \\
\left(\times 10^{-20}\right. \\
\left.\mathrm{m}^{2}\right)\end{array}$ & $\begin{array}{l}\text { p-TCS } \\
\left(\times 10^{-20}\right. \\
\left.\mathrm{m}^{2}\right)\end{array}$ \\
\hline 0.1 & 36.12 & 0 & 0 & 0 & 36.12 \\
\hline 0.15 & 30.52 & 0 & 0 & 0 & 30.52 \\
\hline 0.2 & 26.544 & 0 & 0 & 0 & 26.544 \\
\hline 0.3 & 20.916 & 0 & 0 & 0 & 20.916 \\
\hline 0.4 & 19.348 & 0 & 0 & 0 & 19.348 \\
\hline 0.5 & 18.676 & 0 & 0 & 0 & 18.676 \\
\hline 0.7 & 16.576 & 0 & 0 & 0 & 16.576 \\
\hline 1 & 13.608 & 0 & 0 & 0 & 13.608 \\
\hline 1.5 & 10.248 & 0 & 0 & 0 & 10.248 \\
\hline 2 & 8.316 & 0 & 0 & 0 & 8.316 \\
\hline 3 & 6.356 & 0 & 0 & 0 & 6.356 \\
\hline 4 & 5.404 & 0 & 0 & 0 & 5.404 \\
\hline 5 & 4.844 & 0 & 0 & 0 & 4.844 \\
\hline 7 & 4.06 & 0 & 0 & 0 & 4.06 \\
\hline 10 & 3.108 & 1.34736 & 0 & 0.04704 & 4.508 \\
\hline 15 & 2.5956 & 2.744 & 0.13132 & 2.78068 & 8.26 \\
\hline 20 & 2.996 & 2.296 & 1.5624 & 3.3656 & 10.248 \\
\hline 30 & 3.64 & 1.568 & 3.612 & 2.464 & 11.284 \\
\hline 40 & 3.78 & 1.204 & 4.284 & 1.82 & 11.116 \\
\hline 50 & 3.64 & 0.98 & 4.424 & 1.456 & 10.5 \\
\hline 70 & 3.108 & 0.532 & 4.284 & 1.12 & 9.044 \\
\hline 100 & 2.4808 & 0.224 & 3.864 & 0.896 & 7.476 \\
\hline 150 & 1.8984 & 0.084 & 3.248 & 0.784 & 6.02 \\
\hline 200 & 1.5484 & 0.028 & 2.828 & 0.7 & 5.124 \\
\hline 300 & 1.1452 & 0.028 & 2.2456 & 0.6104 & 4.004 \\
\hline 400 & 0.9184 & 0.0056 & 1.876 & 0.5488 & 3.36 \\
\hline 500 & 0.77 & 0.0028 & 1.6212 & 0.4928 & 2.884 \\
\hline 700 & 0.5852 & 0 & 1.2824 & 0.4144 & 2.282 \\
\hline 1000 & 0.4368 & 0 & 0.9856 & 0.3332 & 1.7584 \\
\hline 2000 & 0.24864 & 0 & 0.5684 & 0.2072 & 1.0248 \\
\hline 3000 & 0.17836 & 0 & 0.406 & 0.1512 & 0.7364 \\
\hline 4000 & 0.14084 & 0 & 0.3164 & 0.1204 & 0.5796 \\
\hline 5000 & 0.11704 & 0 & 0.26068 & 0.10052 & 0.4788 \\
\hline 7000 & 0.08792 & 0 & 0.1932 & 0.07588 & 0.3584 \\
\hline 10000 & 0.06468 & 0 & 0.13944 & 0.05572 & 0.25984 \\
\hline
\end{tabular}

The $\mathrm{O}_{2}$ cross sections (Fig. 2) display much the same characteristics as those of $\mathrm{N}_{2}$ and are reported in Table 2. As for $\mathrm{N}_{2}$, the difference in the $\mathrm{O}_{2}$ electron and positron TCSs at high electron impact energy is more than $20 \%$ up to the limit of our calculation. A small inconsistency is seen in the positronium formation cross section at $200-300 \mathrm{eV}$, where the cross section is very low and seems to be constant. This is an artefact of the method to produce various inelastic cross sections from an initial calculation. In this case the positronium cross section is formed from the subtraction from the inelastic cross section of the excitation and ionisation components, thus the shape can have up to $20 \%$ uncertainty and affect the smoothness of the curve.

As can be seen in Figures 3 and 4, there has been considerable theoretical and experimental work in determining the TCS of positron scattering with both nitrogen and oxygen diatomic molecules. One of the main drawbacks of these comparisons is that the experimental data often fall short of the minimum energy for the accepted TCS data from the Born approximation $(10 \mathrm{keV})$ making comparisons at mid-range to high energies difficult.

In the recent positron scattering database of Brunger et al. [6], the $\mathrm{N}_{2}$ Zecca et al. [17] data were recommended below $40 \mathrm{eV}$, while the $\mathrm{O}_{2}$ Chiari et al. [24] data were recommended below $50 \mathrm{eV}$. At low impact energies $(0.1-10 \mathrm{eV})$ there is a local minimum in the TCS shown in 


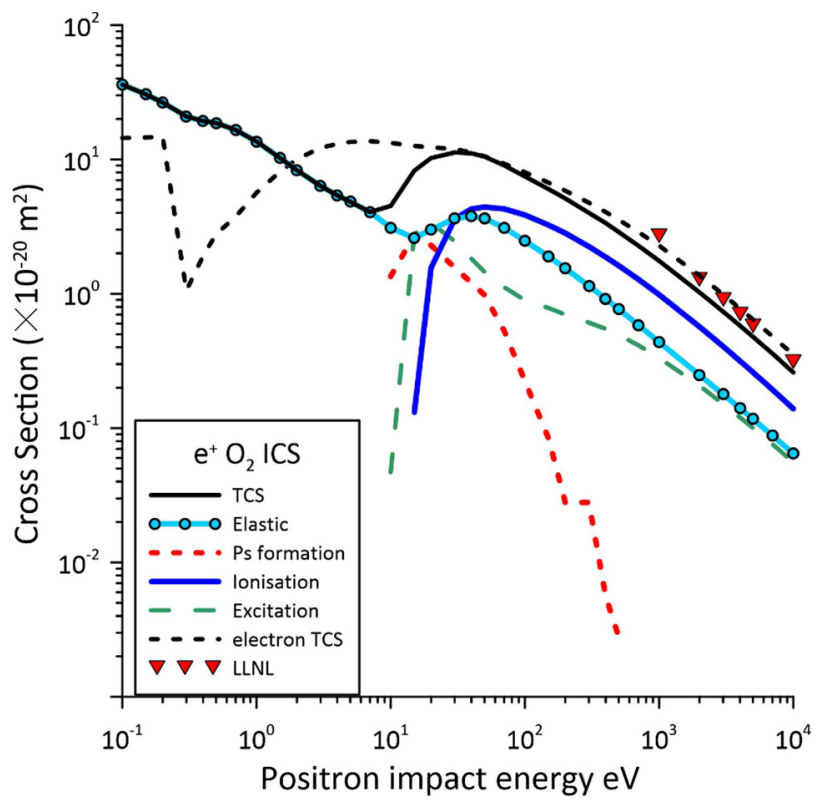

Fig. 2. Integral cross sections for $\mathrm{e}^{+}-\mathrm{O}_{2}$ calculated using the IAM-SCAR+I method. These include elastic and inelastic processes and the $\mathrm{e}^{-}-\mathrm{O}_{2}$ TCS calculated with the same method is shown for comparison. $\mathrm{e}^{-}-\mathrm{O}_{2}$ TCS from the Lawrence Livermore National Laboratory [39] (LLNL) included for comparison.

the Zecca et al. experimental data for $\mathrm{N}_{2}$ and the Chiari et al. experimental data for $\mathrm{O}_{2}$. To model this minimum adequately the choice of the threshold for Ps formation and electronic excitation is vital, along with the elastic scattering cross section. Our calculation of the threshold has been described in the calculation section. For $\mathrm{N}_{2}$ the IAM-SCAR+I method gives a minimum at $10 \mathrm{eV}$, and Zecca et al. indicate it at $6.35 \pm 0.1 \mathrm{eV}$. With the IAM-SCAR+I for $\mathrm{O}_{2}$ the minimum is $7 \mathrm{eV}$ compared to $3.0 \pm 0.05 \mathrm{eV}$ from Chiari et al. With more data points calculated at low energies, this minimum could be more accurately modelled with IAM-SCAR+I. At energies above the minimum, the IAM-SCAR $+\mathrm{I}$ calculation is coincident with experimental (and some, but not all calculation) data for several $\mathrm{eV}$ however below this we overestimate the TCS by approx. $70 \%$ for $\mathrm{N}_{2}$ and $220 \%$ for $\mathrm{O}_{2}$. Notably the slope below the local minima near $10 \mathrm{eV}$ is similar between the IAM-SCAR + I data and the recommended data. This can be attributed to the elastic atomic potentials used at these low energies. As can be seen from both Figures 3 and 4, the other calculation methods either do not attempt to model this difficult area, or they show similar discrepancies with the available data.

In the low - mid energy region $(10-100 \mathrm{eV})$ the IAMSCAR + I method lies above all experimental and theoretical data. Above $40 \mathrm{eV}$ the Hoffman et al. [18] data were recommended in the Brunger et al. [6] database for $\mathrm{N}_{2}$ and this data set sits $20-30 \%$ below the calculated IAM-SCAR $+\mathrm{I}$ data for $\mathrm{N}_{2}$. This overestimation is even clearer for the case of $\mathrm{O}_{2}$ in Figure 4. The $\mathrm{O}_{2}$ calculated TCS has a maximum discrepancy to the experimental data (the Chiari experiments [24], recommended) of $53 \%$,

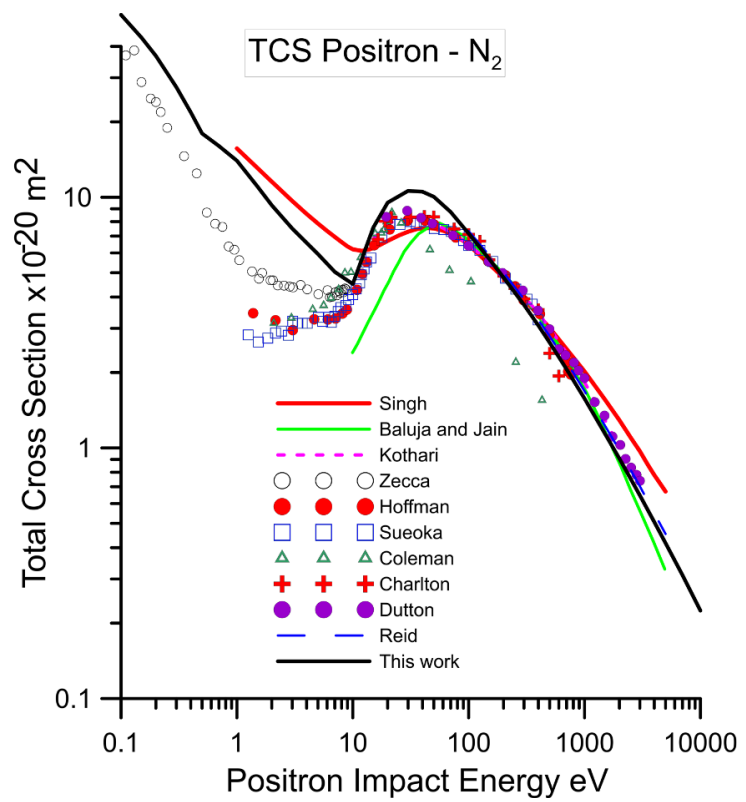

Fig. 3. $\mathrm{N}_{2}$ molecule total cross sections from a variety of experimental and calculated methods compared to the IAMSCAR $+\mathrm{I}$ results of this study for the positron impact energy range 0.1 to $10000 \mathrm{eV}[16-19,44-48]$.

at $50 \mathrm{eV}$, where the experimental uncertainty was in the $5-13 \%$ range. Above $50 \mathrm{eV}$ the Charlton et al. [46] data are recommended and appears convergent with IAM-SCAR+I at the highest energy of $600 \mathrm{eV}$.

Many of the experimental papers discuss the angular discrimination and its pertinent effect of lowering the TCS due to the increased acceptance of elastically scattered positrons, and this can in some way reduce the discrepancy seen. For the data taken by Zecca et al. [17] and Chiari et al. [24] at the facility in Trento, the angular discrimination at $50 \mathrm{eV}$ misses those angles below $2.4^{\circ}$ [17]. This missing angle increases as the impact energy decreases, to $17.5^{\circ}$ at $1 \mathrm{eV}$. The Hoffman et al. [18] $\mathrm{N}_{2}$ data, on the other hand, have an angular discrimination estimated up to $21^{\circ}$ at $50 \mathrm{eV}$, and fluctuates between $16^{\circ}$ and $22^{\circ}$ across their energy range. The Charlton et al. [46] $\mathrm{O}_{2}$ publication does not mention the angular discrimination of their apparatus. Some of the discrepancies between the IAM-SCAR+I calculation and the experimental data can be attributed to this angular discrimination; however this would reduce, rather that remove, the differences seen. We should note here that this discrepancy does not occur in the case of the electron scattering. We have recently shown [52] how our IAM-SCAR + I calculation of the electron scattering TCS for $\mathrm{N}_{2}$ perfectly agrees with our own measurements and with the recommended data from Itikawa [53] in the 10-100 energy range.

The low-mid energy region is also where a comparison of inelastic processes can be made between experiment and theory. In Figure 5 we compare the positronium formation and ionisation cross sections of Marler and Surko [43] to the IAM-SCAR+I calculation for $\mathrm{N}_{2}$. In Figure 6 we compare the $\mathrm{O}_{2}$ ionisation cross sections of Marler and Surko [43] to the IAM-SCAR+I method, and the 


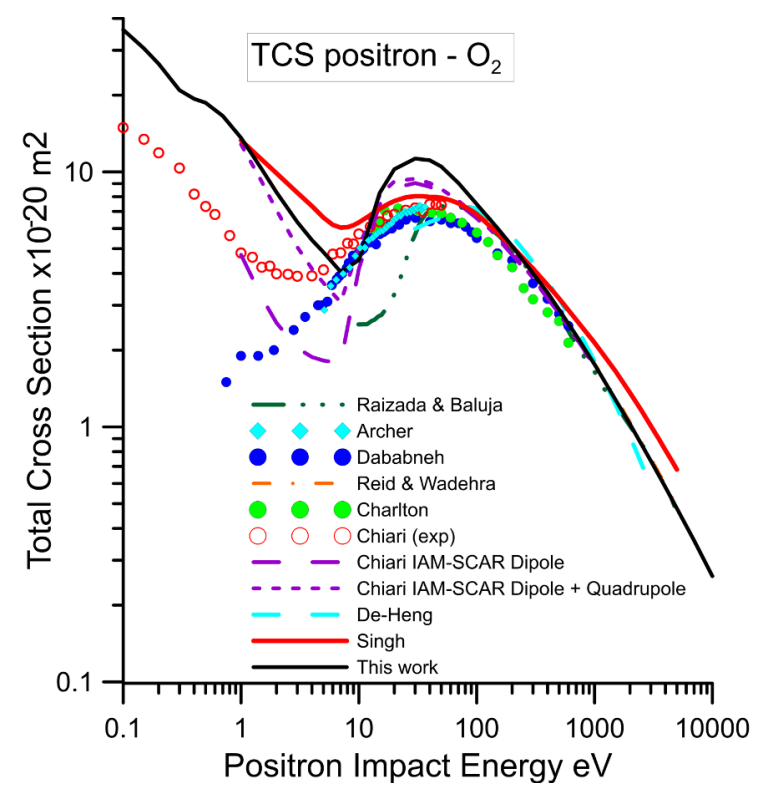

Fig. 4. $\mathrm{O}_{2}$ molecule total cross sections from a variety of experimental and calculated methods to compare to our IAM$\mathrm{SCAR}+\mathrm{I}$ results for the positron impact energy range 0.1 to $10000 \mathrm{eV}[10,24,46,47,49-51]$ including previous IAM-SCAR results from 2012 utilising the dipole or dipole plus quadrupole formalism of the atomic polarization potentials (Eq. (1)), but without interference effects.

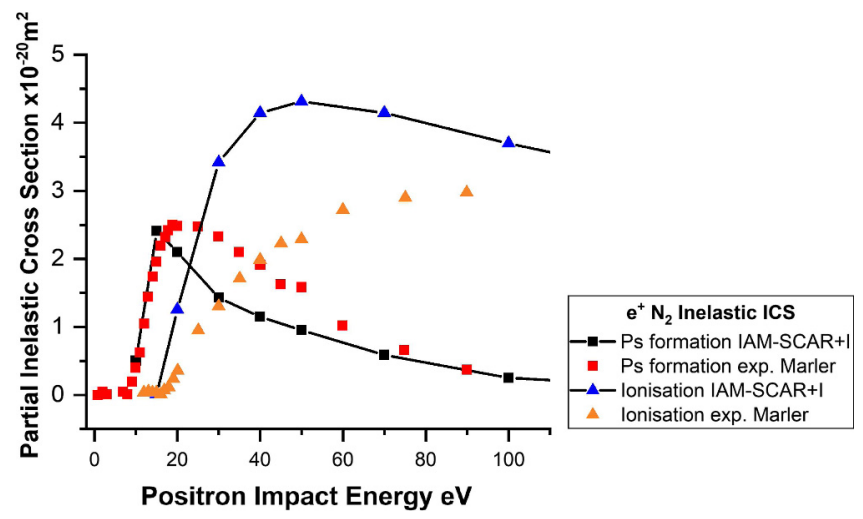

Fig. 5. Positron- $\mathrm{N}_{2}$ inelastic partial cross sections comparing experiments from Marler and Surko [43] to the IAM-SCAR+I calculations. Black lines with scatter points indicate the calculation, only scatter points indicate experiment. Squares show positronium formation and triangles show ionisation.

positronium formation cross sections of both Marler and Surko [43], and Archer et al. [50] to the IAM-SCAR+I method. Marler and Surko [43] used a cold trapped positron beam and a gas cell, utilizing a strong magnetic field and a retarding potential analyser, ensuring a high collection efficiency and that the kinetic energy of the positrons is detected appropriately. Positronium formation was detected by loss of charged particles from the beam, and ionisation was detected by limiting detection to those particles that have lost the equivalent to the ionisation limit or more in kinetic energy. Archer et al. [50] used

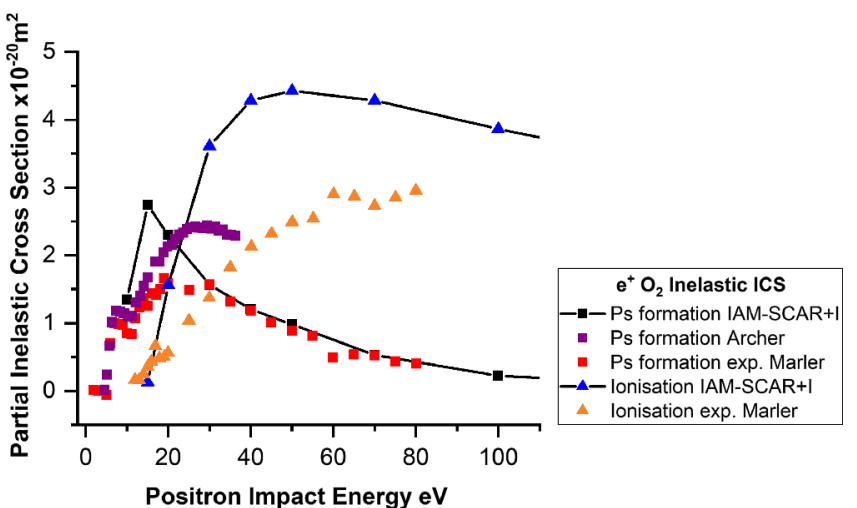

Fig. 6. Positron- $\mathrm{O}_{2}$ inelastic partial cross sections comparing experiments from Marler and Surko [43] and Archer et al. [50] to the IAM-SCAR+I calculations. Black lines with scatter points indicate the calculation, only scatter points indicate experiment. Squares show positronium formation and triangles show ionisation.

a similar experiment, though without trapping, directing their positron beam again through a gas cell and determining the positronium formation through the beam intensity loss.

The IAM-SCAR+I calculated ionisation integral partial cross sections are slightly higher than the experimental values [43], as seen clearly in Figures 5 and 6 for both $\mathrm{N}_{2}$ and $\mathrm{O}_{2}$ (triangles in both spectra). This discrepancy, being near $1.5 \times 10^{-20} \mathrm{~m}^{2}$, is a narrow gap and the causes can be difficult to determine. The calculation shows a maxima around $50 \mathrm{eV}$ energy, and exhibits a standard shape for an ionisation cross section. For the experimental data for both $\mathrm{O}_{2}$ and $\mathrm{N}_{2}$ no maxima are seen for the ionisation cross sections up to their limit of $90 \mathrm{eV}$.

The positronium formation has a better fit between the experiment and the calculation. In the case of nitrogen (Fig. 5), the experiment is slightly above the calculated values, the reverse of what was seen for ionisation cross sections for both molecules. The difference is small- $1 \times$ $10^{-20} \mathrm{~m}^{2}$. In the case of oxygen (Fig. 6), the values of the integral partial cross section for positronium formation coincide from $30 \mathrm{eV}$, and the calculation has higher values than the Marler and Surko experiment [43] but lower values than the Archer [50] experiment. The variability in the two sets of experimental values essentially bracket the IAM-SCAR+I calculated values. One notable feature is that the peak in the positronium formation cross section for the IAM-SCAR $+\mathrm{I}$ calculation is a few $\mathrm{eV}$ below the experiment. The level of accuracy achieved for the IAM-SCAR+I model for individual inelastic partial cross sections is particularly astounding considering they arise from a subtraction process outlined in the methods. This process also accounts for the missing feature seen in the experimental $\mathrm{O}_{2}$ positronium formation at $7 \mathrm{eV}$, which has been attributed to the onset of the Schumann-Runge continuum in $\mathrm{O}_{2}[43,52]$.

In the mid to high energy range $(100-10000 \mathrm{eV})$ it is seen in Figures 3 and 4 that the data we present fit well to the existing experimental and calculation work, save for that of Singh et al. [10], which appears to diverge above 


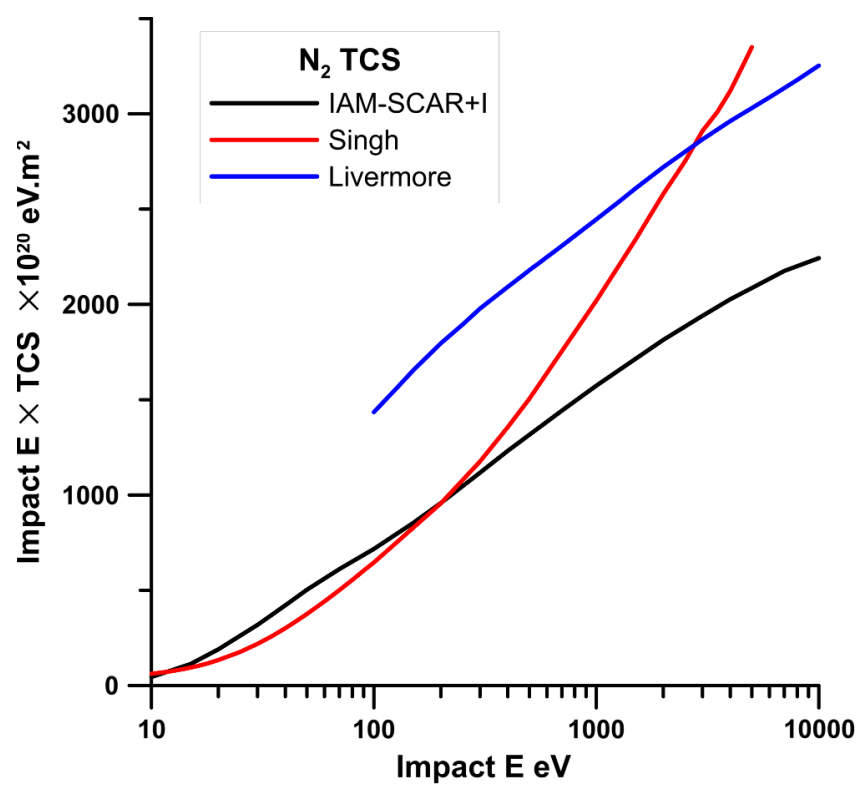

Fig. 7. A "Fano" plot, of E. $\sigma$ vs log E for the positron scattering TCS of $\mathrm{N}_{2}$ from the calculation methods used in this work (black), that of Singh et al. (red) and the well known Livermore database values for electron scattering TCS as a comparison for shape (blue).

$300 \mathrm{eV}$ for the oxygen and nitrogen total cross section datasets. This fit is of primary importance when assessing the validity of cross sections at high energies and can be more easily shown in the Fano plots of Figures 7 and 8, where we also include data from the Livermore database for electron scattering of $\mathrm{O}_{2}$ and $\mathrm{N}_{2}$ using the additivity rule. Validity to the Born approximation (at higher energies) requires the plot of E.TCS vs $\log \mathrm{E}$ tend to be linear for increasing energies. This is a feature of the Born approximation formalism of the total cross section, where the slope tends towards the oscillator strength when plotted under the correct units [54]. As detailed in a previous paper [24], the positron scattering cross section resides lower than the electron scattering cross section at energies up to $10 \mathrm{keV}$ due to the different polarity of the terms in the real part of the optical potential. As such, comparison to electron scattering for these energies is no longer quantitatively viable, however the shape of the two curves can be compared. As is seen in Figures 7 and 8, the IAM-SCAR+I method delivers on this linearity, while the SCOP method proposed by Singh indicates a non-linear relationship. Additionally, we expect convergence of the positron scattering cross sections with the slope of the electron cross section trend above approx. $100 \mathrm{eV}$. As can be seen our positron calculations for nitrogen fulfil this requirement, having a difference in slope of $<12 \%$ to the Livermore data, compared to $86 \%$ for that from Singh et al. Oxygen fares better, with a slope less than $1 \%$ different to the Livermore data, whereas the slope of the Singh data is $75 \%$ sharper than both.

As Singh et al. [10] also use the optical potential method to compute their TCS, the reason for this discrepancy at high energy and the unexpected angular dependence

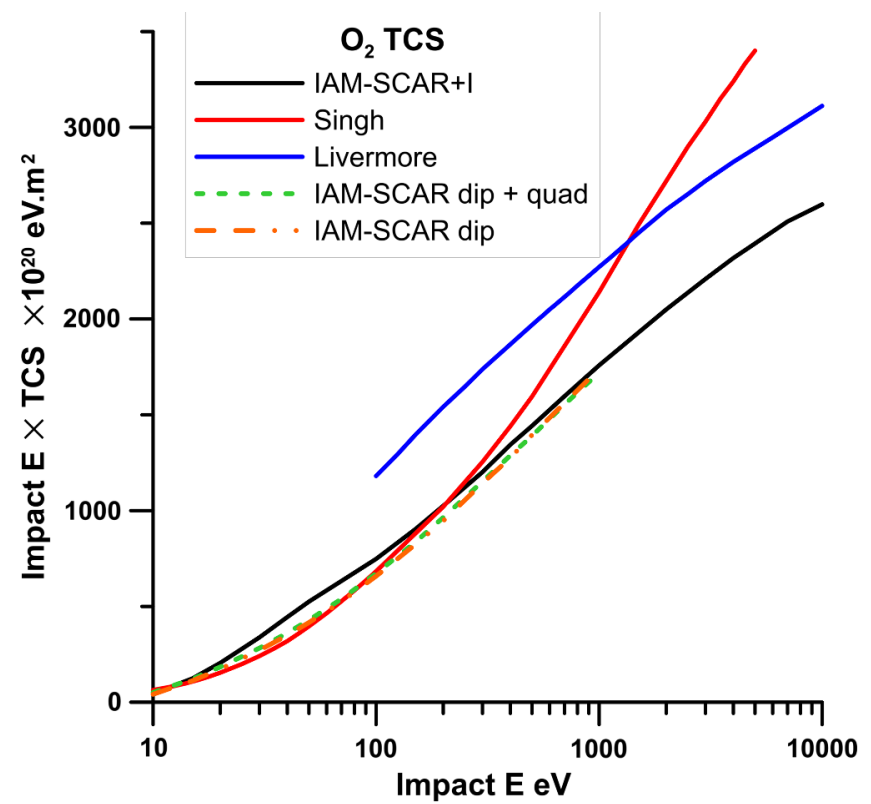

Fig. 8. A "Fano" plot, of E. $\sigma$ vs log E for the positron scattering TCS of $\mathrm{O}_{2}$ from the calculation methods used in this work (black), that of Singh et al. (red) and the well known Livermore database values for electron scattering TCS as a comparison for shape (blue). Additionally two previous formulations of the IAM-SCAR positron scattering method are shown (green, red), indicating the similar convergence to the normal behavior.

with respect to the Born-Bethe theory Fano plots in Figures 7 and 8 may arise from their treatment of the molecule geometry. They state that "the charge density and static potential is obtained by expanding the respective functions from the center of mass of the molecule." . This will by necessity overestimate the cross sections as it does not remove the "screened" areas of the cross sections occurring when using the additivity rule for energies below $1000 \mathrm{eV}$ [55]. At the highest energy available from the Singh method $(5000 \mathrm{eV})$ their TCS even exceed those of the Livermore database, where the simple additivity rule is used to give the diatomic TCS. As such the potentials used may add to this discrepancy in their case. It is clear to us that while their method is closer to experimental values between 10 and $100 \mathrm{eV}$, nicely processing the maxima due to the inelastic processes, beyond this the models differ significantly.

At high and low impact energies across the calculated range, the IAM-SCAR+I method reproduces the literature data well for positron impact with oxygen and nitrogen. The overestimation of the IAM-SCAR $+\mathrm{I}$ calculation for positrons in the region where the maxima of inelastic scattering resides, in both cases, can indicate several things. As mentioned, some of this discrepancy may be due to the experimental data indicating a lower boundary on the total cross sections [24] due to the angular discrimination. It is likely, however, that elements of the formalisms used do not comply well with the reality of the scattering situation, in the case of positrons. As the positronium formation is quite relevant in the mentioned $10-100 \mathrm{eV}$ energy range, this seems to indicate that the 
description of this process within our IAM-SCAR procedure needs some improvements. As noted, these issues are not seen for the new formalism when calculating cross sections for electron impact.

\section{Conclusions}

We present new $\mathrm{N}_{2}$ and updated $\mathrm{O}_{2}$ positron scattering integral cross section data calculated using the recently improved IAM-SCAR+I method for impact positron energies of $0.1-10000 \mathrm{eV}$. Modifications to our calculation method have improved the behavior at and below the positronium formation threshold and above $100 \mathrm{eV}$, though there are still improvements to be made in calculating the $10-100 \mathrm{eV}$ region where the inelastic processes are most relevant. The interference effect, while bringing our calculations in line with the full optical potential method and including more realistic processes, enhances the importance of accurate atomic scattering potentials. We compare our results to the breadth of experimental and calculated results available in the literature, with particular emphasis on a new formalism published in 2016 [10]. This comparison finds the IAM-SCAR+I to outperform the new formalism above $100 \mathrm{eV}$ and below $10 \mathrm{eV}$, however the mentioned issues at intermediate energies will be addressed in future work.

We acknowledge the financial support received from the European Union Seventh Framework Programme (ITNARGENT Marie Curie project), under grant agreement No. 608163, and from the Spanish Ministerio de Economia y Competitividad (project No. FIS2016-80440).

\section{Author contribution statement}

F.B. and G.G. developed the IAM-SCAR+I calculation method. G.G. conceived and planned the calculation. F.B. prepared the calculation files. L.E.-G. analysed the data and outlined the context within the field. L.E.-G. wrote the manuscript with support from G.G.

Open Access This is an open access article distributed under the terms of the Creative Commons Attribution License (http://creativecommons.org/licenses/by/4.0/), which permits unrestricted use, distribution, and reproduction in any medium, provided the original work is properly cited.

\section{References}

1. A. Muñoz, D. Almeida, F. Ferreira da Silva, P. LimãoVieira, M.C. Fuss, A.G. Sanz, G. García, Eur. Phys. J. D 67, 1 (2013)

2. D.L. Bailey, D.W. Townsend, P.E. Valk, M.N. Maisey, editors, Positron Emission Tomography (Springer-Verlag, London, 2005)
3. S.E. Combs, J. Bauer, D. Unholtz, C. Kurz, T. Welzel, D. Habermehl, T. Haberer, J. Debus, K. Parodi, BMC Cancer 12, 133 (2012)

4. R.P. McEachran, J.P. Sullivan, S.J. Buckman, M.J. Brunger, M.C. Fuss, A. Muñoz, F. Blanco, R.D. White, Z.L. Petrović, P. Limão-Vieira, G. García, J. Phys. B At. Mol. Opt. Phys. 45, 045207 (2012)

5. A. Muñoz, M.C. Fuss, M.A. Cortés-Giraldo, S. Incerti, V. Ivanchenko, A. Ivanchenko, J.M. Quesada, F. Salvat, C. Champion, G. García Gómez-Tejedor, in Radiation damage in biomolecular systems, edited by G. García Gómez-Tejedor, M.C. Fuss (Springer, Netherlands, 2012), pp. 203-225

6. M.J. Brunger, S.J. Buckman, K. Ratnavelu, J. Phys. Chem. Ref. Data 46, 023102 (2017)

7. P. Coleman, Positron Beams and Their Applications (World Scientific, 2000)

8. F. Blanco, A.M. Roldán, K. Krupa, R.P. McEachran, R.D. White, S. Marjanović, Z.L. Petrović, M.J. Brunger, J.R. Machacek, S.J. Buckman, J.P. Sullivan, L. Chiari, P. Limão-Vieira, G. García, J. Phys. B At. Mol. Opt. Phys. 49, 145001 (2016)

9. S. Schippers, E. Sokell, F. Aumayr, H. Sadeghpour, K. Ueda, I. Bray, K. Bartschat, A. Murray, J. Tennyson, A. Dorn, M. Yamazaki, M. Takahashi, N. Mason, O. Novotný, A. Wolf, L. Sanche, M. Centurion, Y. Yamazaki, G. Laricchia, C.M. Surko, J. Sullivan, G. Gribakin, D.W. Savin, Y. Ralchenko, R. Hoekstra, G. O'Sullivan, J. Phys. B At. Mol. Opt. Phys. 52, 171002 (2019)

10. S. Singh, S. Dutta, R. Naghma, B. Antony, J. Phys. Chem. A 120, 5685 (2016)

11. F. Blanco, G. García, Chem. Phys. Lett. 635, 321 (2015)

12. F. Blanco, L. Ellis-Gibbings, G. García, Chem. Phys. Lett. 645, 71 (2016)

13. D.B. Jones, R.F. da Costa, M.D.N. Varella, M.H.F. Bettega, M.A.P. Lima, F. Blanco, G. García, M.J. Brunger, J. Chem. Phys. 144, 144303 (2016)

14. F. Blanco, G. García, Phys. Rev. A 67, 022701 (2003)

15. F. Blanco, G. García, Phys. Lett. A 330, 230 (2004)

16. H.N. Kothari, K.N. Joshipura, Pramana J. Phys. 76, 477 (2011)

17. A. Zecca, L. Chiari, A. Sarkar, M.J. Brunger, New J. Phys. 13, 115001 (2011)

18. K.R. Hoffman, M.S. Dababneh, Y.F. Hsieh, W.E. Kauppila, V. Pol, J.H. Smart, T. S. Stein, Phys. Rev. A 25, 1393 (1982)

19. O. Sueoka, S. Mori, J. Phys. Soc. Japan 53, 2491 (1984)

20. A.G. Sanz, M.C. Fuss, F. Blanco, Z. Mašín, J.D. Gorfinkiel, F. Carelli, F. Sebastianelli, F.A. Gianturco, G. García, Appl. Radiat. Isot. 83, 57 (2014)

21. F. Blanco, G. García, J. Phys. B At. Mol. Opt. Phys. 42, $145203(2009)$

22. F. Blanco, J. Rosado, A. Illana, G. García, Phys. Lett. A 374, 4420 (2010)

23. H. Kato, K. Anzai, T. Ishihara, M. Hoshino, F. Blanco, G. García, P. Limão-Vieira, M.J. Brunger, S.J. Buckman, H. Tanaka, J. Phys. B At. Mol. Opt. Phys. 45, 095204 (2012)

24. L. Chiari, A. Zecca, S. Girardi, E. Trainotti, G. García, F. Blanco, R.P. McEachran, M.J. Brunger, J. Phys. B At. Mol. Opt. Phys. 45, 215206 (2012)

25. A. Muñoz, J.C. Oller, F. Blanco, J.D. Gorfinkiel, P. Limão-Vieira, A. Maira-Vidal, M.J.G. Borge, O. Tengblad, C. Huerga, M. Téllez, G. García, J. Phys. Conf. Ser. 133, 012002 (2008) 
26. P. Palihawadana, J. Sullivan, M. Brunger, C. Winstead, V. McKoy, G. García, F. Blanco, S. Buckman, Phys. Rev. A 84, 62702 (2011)

27. A. Zecca, L. Chiari, G. García, F. Blanco, E. Trainotti, M.J. Brunger, J. Phys. B At. Mol. Opt. Phys. 43, 215204 (2010)

28. A.G. Sanz, M.C. Fuss, F. Blanco, Z. Mašín, J.D. Gorfinkiel, R.P. McEachran, M.J. Brunger, G. García, Phys. Rev. A 88, 062704 (2013)

29. D. Stevens, T.J. Babij, J.R. Machacek, S.J. Buckman, M.J. Brunger, R.D. White, G. García, F. Blanco, L. Ellis-Gibbings, J.P. Sullivan, J. Chem. Phys. 148, (2018)

30. F. Blanco, G. García, Phys. Lett. A 360, 707 (2007)

31. G. Garcia, F. Blanco, A. Williart, Chem. Phys. Lett. 335, 227 (2001)

32. L. Chiari, E. Anderson, W. Tattersall, J.R. Machacek, P. Palihawadana, C. Makochekanwa, J.P. Sullivan, G. García, F. Blanco, R.P. McEachran, M.J. Brunger, S.J. Buckman, J. Chem. Phys. 138, 074301 (2013)

33. D.D. Reid, J.M. Wadehra, J. Phys. B At. Mol. Opt. Phys. 29, L127 (1996)

34. R.P. McEachran, A.G. Ryman, A.D. Stauffer, D.L. Morgan, J. Phys. B At. Mol. Phys. 10, 663 (1977)

35. A.C.L. Jones, C. Makochekanwa, P. Caradonna, D.S. Slaughter, J.R. Machacek, R.P. McEachran, J.P. Sullivan, S.J. Buckman, A.D. Stauffer, I. Bray, D.V. Fursa, Phys. Rev. A 83, 032701 (2011)

36. E.-A. Reinsch, W. Meyer, Phys. Rev. A 18, 1793 (1978)

37. H.-J. Werner, W. Meyer, Phys. Rev. A 13, 13 (1976).

38. PubChem, PubChem Database (2019)

39. S.T. Perkins, D.E. Cullen, S.M. Seltzer, Tables and Graphs of Electron-Interaction Cross Sections from $10 \mathrm{EV}$ to 100 GeV Derived from the LLNL Evaluated Electron Data Library (EEDL), $Z=1-100$ (1991)
40. P. Chaudhuri, M.T.D.N. Varella, C.R.C. de Carvalho, M. A.P. Lima, Nucl. Instrum. Meth. Phys. Res. Sect. B Beam Interact. Mater. At. 221, 69 (2004)

41. J.P. Sullivan, J.P. Marler, S.J. Gilbert, S.J. Buckman, C.M. Surko, Phys. Rev. Lett. 87, 073201 (2001)

42. W. Sun, M.A. Morrison, W.A. Isaacs, W.K. Trail, D.T. Alle, R.J. Gulley, M.J. Brennan, S.J. Buckman, Phys. Rev. A 52, 1229 (1995)

43. J.P. Marler, C.M. Surko, Phys. Rev. A At. Mol. Opt. Phys. 72, 1 (2005)

44. P.G. Coleman, T.C. Griffith, G. R. Heyland, Appl. Phys. 4, 89 (1974)

45. K.L. Baluja, A. Jain, Phys. Rev. A 45, 7838 (1992)

46. M. Charlton, T.C. Griffith, G.R. Heyland, G.L. Wright, J. Phys. B At. Mol. Phys. 13, L353 (1980)

47. D.D. Reid, J.M. Wadehra, Chem. Phys. Lett. 311, 385 (1999)

48. J. Dutton, C.J. Evans, H.L. Mansour, J. Phys. B At. Mol. Phys. 20, 2607 (1987)

49. S. De-Heng, L. Yu-Fang, S. Jin-Feng, Z. Zun-Lue, Y. Xiang-Dong, Chinese Phys. 14, 964 (2005)

50. J. Archer, S.M. Trilov, P.G. Coleman, J. Phys. Conf. Ser. 443, 012001 (2013)

51. R. Raizada, K.L. Baluja, Phys. Rev. A 55, 1533 (1997)

52. G. Laricchia, J. Moxom, M. Charlton, Phys. Rev. Lett. 70, 3229 (1993)

53. A.I. Lozano, J.C. Oller, K. Krupa, F. Ferreira da Silva, P. Limão-Vieira, F. Blanco, A. Muñoz, R. Colmenares, G. García, Rev. Sci. Instrum. 89, 063105 (2018)

54. Y. Itikawa, J. Phys. Chem. Ref. Data 35, 31 (2006)

55. U. Fano, A.R.P. Rau, in Atomic Collisions and Spectra (1986), pp. 51-54

56. F. Blanco, G. Garcia, Phys. Lett. A 317, 458 (2003) 